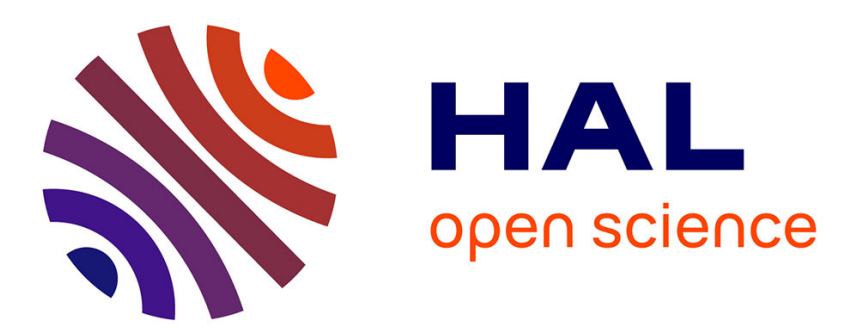

\title{
Proof Pearl: Constructive Extraction of Cycle Finding Algorithms
}

Dominique Larchey-Wendling

\section{To cite this version:}

Dominique Larchey-Wendling. Proof Pearl: Constructive Extraction of Cycle Finding Algorithms. 9th International Conference on Interactive Theorem Proving, ITP 2018, Jul 2018, Oxford, United Kingdom. pp.370-387, 10.1007/978-3-319-94821-8_22 . hal-02333354

\section{HAL Id: hal-02333354 https://hal.science/hal-02333354}

Submitted on 9 Nov 2020

HAL is a multi-disciplinary open access archive for the deposit and dissemination of scientific research documents, whether they are published or not. The documents may come from teaching and research institutions in France or abroad, or from public or private research centers.
L'archive ouverte pluridisciplinaire HAL, est destinée au dépôt et à la diffusion de documents scientifiques de niveau recherche, publiés ou non, émanant des établissements d'enseignement et de recherche français ou étrangers, des laboratoires publics ou privés. 


\title{
Proof Pearl: Constructive Extraction of Cycle Finding Algorithms
}

\author{
Dominique Larchey-Wendling \\ Université de Lorraine, CNRS, LORIA, Nancy, France \\ dominique. larchey-wendling@loria.fr
}

\begin{abstract}
We present a short implementation of the well-known Tortoise and Hare cycle finding algorithm in the constructive setting of Coq. This algorithm is interesting from a constructive perspective because it is both very simple and potentially non-terminating (depending on the input). To overcome potential non-termination, we encode the given termination argument (there exists a cycle) into a bar inductive predicate that we use as termination certificate. From this development, we extract the standard OCaml implementation of this algorithm. We generalize the method to the full Floyd's algorithm that computes the entry point and the period of the cycle in the iterated sequence, and to the more efficient Brent's algorithm for computing the period only, again with accurate extractions of their respective standard OCaml implementations.
\end{abstract}

Keywords: Cycle finding $\cdot$ Bar inductive predicates $\cdot$ Partial algorithms in Coq $\cdot$ Correctness by extraction

\section{Introduction}

The Tortoise and the Hare (T\&H for short) in particular and cycle detection [1] in general are standard algorithms that will very likely cross the path of any wouldbe computer scientist. They aim at detecting cycles in deterministic sequences of values, i.e. when the next value depends only on the current value. They have many applications, from pseudorandom number strength measurement, integer factorization through Pollard's rho algorithm [18] or more generally cryptography, etc, even celestial mechanics. But our interest with those algorithms lies more in the framework in which we want to implement and certify them:

- first we want to prove the partial correction of cycle detection algorithms without assuming their termination. Hence, we do not restrict our study to finite domains. In the finitary case indeed, the pigeon hole principle ensures that there is always a cycle to detect and termination can be certified by cardinality considerations 9 ;

- inductive/constructive type theories constitute challenging contexts for these algorithms because they are inherently partial. The reason for that is the undecidability of the existence of a cycle in an arbitrary given sequence. Hence, we need to work with partial recursive functions;

Work partially supported by the TICAMORE project (ANR grant 16-CE91-0002). 
- Coq could wrongfully be considered being limited to total functions. To work around this, Hilbert's $\epsilon$-operator (a non-constructive form of the axiom of choice) is sometimes postulated as a convenient way to deal with partial functions [7]. Why not, HOL is based on it? We argue that we can stay fully constructive and we will show that axiom-free Coq can work with such partial recursive functions provided they are precisely specified.

T\&H was attributed to Robert W. Floyd by Donald E. Knuth [15] but may in fact be a folk theorem (see [2, footnote 8 on page 21). The idea is to launch from a starting point $x_{0}$ both a slow tortoise (the tortoise steps once at each iteration) and a quick hare (the hare steps twice at each iteration). Then the hare will recapture the tortoise if and only if there is a cycle in the sequence from $x_{0}$. We refer to [1] for further visual explanations about the origin and intuition behind this very well-known algorithm. The T\&H algorithm computes only a meeting point for the two fabulous animals. We will call Floyd's cycle finding the algorithm that builds on this technique to compute the entry point and period of the cycle. We also consider Brent's period only finding algorithm [6] that proceeds with a slow hare and a so-called "teleporting tortoise."

Because these algorithms do not always terminate, defining the corresponding fully specified Coq fixpoints might be considered challenging. The folklore fuel trick could be used to simulate general recursion in Coq. The idea is to use a term of the type $X \rightarrow$ (fuel : nat) $\rightarrow$ option $Y$ to represent a partial recursive function $f: X \rightarrow Y$. The fuel argument ensures termination, as e.g. a bound on the number of recursive sub-calls. This fuel trick has several problems: computing a big-enough fuel value from the input $x: X$ might be as complicated as showing the termination of $f(x)$ itself; but also, the fuel argument is informative and is thus preserved by extraction, both as a parasitic argument and as a companion program for computing the input value of fuel from the value of $x$; and finally, the output type is now option $Y$ instead of $Y$ so an extra match construct is necessary. We will show how to replace the informative fuel argument with a non-informative bar inductive predicate to ensure termination. As such, it is erased by extraction, getting us rid of parasitic arguments. In particular, we obtain an OCaml extraction of T\&H certified by less than 80 lines of Coq code.

\section{Formalization of the problem}

Given a set $X$ and a function $f: X \rightarrow X$, we define the $n$-th iterate $f^{n}: X \rightarrow X$ of $f$ by induction on $n: f^{0}=x \mapsto x$ and $f^{n+1}=f \circ f^{n}$. In Coq, this definition corresponds to the code of the iterator (with a convenient compact $f^{n}$ notation):

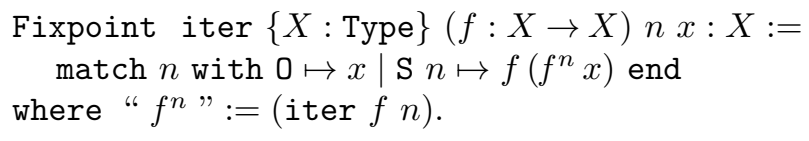

We get the identity $f^{a+b}(x)=f^{a}\left(f^{b}(x)\right)$ by induction on $a$. Given a starting point $x_{0} \in X$, we consider the infinite sequence $x_{0}, f\left(x_{0}\right), f^{2}\left(x_{0}\right), \ldots, f^{n}\left(x_{0}\right), \ldots$ 
of iterates of $f$ on $x_{0}$, i.e. the map $n \mapsto f^{n}\left(x_{0}\right)$. From a classical logic perspective, two mutually exclusive alternatives are possible:

A1 the sequence $n \mapsto f^{n}\left(x_{0}\right)$ is injective, i.e. $f^{i}\left(x_{0}\right) \neq f^{j}\left(x_{0}\right)$ holds unless $i=j$. In this case, there is no cycle in the iterated sequence from $x_{0}$;

A2 there exist $i \neq j$ such that $f^{i}\left(x_{0}\right)=f^{j}\left(x_{0}\right)$ and in this case, there is a cycle in the iterated sequence from $x_{0}$.

It is however not possible to computationally distinguish those two cases: no cycle finding algorithm can be both correct and always terminating. To show this undecidability result, one can reduce the Halting problem to the cycle detection problem (see file cycle_undec.v).

The T\&H algorithm terminates exactly when Alternative A2 above holds and loops forever when Alternative A1 holds. There are many equivalent characterizations of the existence of a cycle, which we call the cyclicity property.

Proposition 1 (Cyclicity). For any set $X$, any function $f: X \rightarrow X$ and any $x_{0} \in X$, the four following conditions are equivalent:

1. there exist $i, j \in \mathbb{N}$ such that $i \neq j$ and $f^{i}\left(x_{0}\right)=f^{j}\left(x_{0}\right)$;

2. there exist $\lambda, \mu \in \mathbb{N}$ such that $0<\mu$ and $f^{\lambda}\left(x_{0}\right)=f^{\lambda+\mu}\left(x_{0}\right)$;

3. there exist $\lambda, \mu \in \mathbb{N}$ s.t. $0<\mu$ and for any $i, j \in \mathbb{N}, f^{i+\lambda}\left(x_{0}\right)=f^{i+\lambda+j \mu}\left(x_{0}\right)$;

4. there exists $\tau \in \mathbb{N}$ such that $0<\tau$ and $f^{\tau}\left(x_{0}\right)=f^{2 \tau}\left(x_{0}\right)$.

Proof. For $1 \Rightarrow 2$, if $i<j$ then choose $\lambda=i$ and $\mu=j-i$ (exchange $i$ and $j$ if otherwise $j<i$ ). For $2 \Rightarrow 3$, first show $f^{\lambda}\left(x_{0}\right)=f^{\lambda+j \mu}\left(x_{0}\right)$ by induction on $j$. Then $f^{i+\lambda}\left(x_{0}\right)=f^{i}\left(f^{\lambda}\left(x_{0}\right)\right)=f^{i}\left(f^{\lambda+j \mu}\left(x_{0}\right)\right)=f^{i+\lambda+j \mu}\left(x_{0}\right)$. For $3 \Rightarrow 4$, choose $\tau=(1+\lambda) \mu$ and derive $f^{\tau}\left(x_{0}\right)=f^{2 \tau}\left(x_{0}\right)$ using $i=(1+\lambda) \mu-\lambda$ and $j=1+\lambda$. For $4 \Rightarrow 1$, choose $i=\tau$ and $j=2 \tau$. That proof is mechanized as Proposition cyclicity_prop in file utils.v.

The functional specification of $\mathrm{T \& H}$ is to compute a meeting index $\tau \in \mathbb{N}$ such that $0<\tau$ and $f^{\tau}\left(x_{0}\right)=f^{2 \tau}\left(x_{0}\right)$ (corresponding to Item 4 of Proposition 1), provided such a value exists. Operationally, the algorithm consists in enumerating the sequence of pairs $\left(f\left(x_{0}\right), f^{2}\left(x_{0}\right)\right),\left(f^{2}\left(x_{0}\right), f^{4}\left(x_{0}\right)\right), \ldots,\left(f^{n}\left(x_{0}\right), f^{2 n}\left(x_{0}\right)\right), \ldots$ in an efficient way until the two values $f^{n}\left(x_{0}\right)$ and $f^{2 n}\left(x_{0}\right)$ are equal.

\subsection{An OCaml account of the Tortoise and the Hare}

The $\mathbf{T} \& \mathrm{H}$ algorithm can be expressed in OCaml as the following two functions:

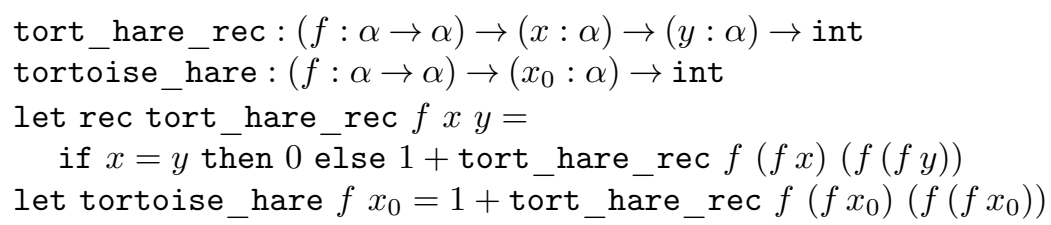

In general, the tail-recursive version is preferred because tail-recursive functions can be compiled into loops without the help of a stack. The code of the function 
tortoise_hare_tail contains a sub-function loop where the first argument $f$ of tortoise_hare_tail is fixed and second argument $x_{0}$ is unused.

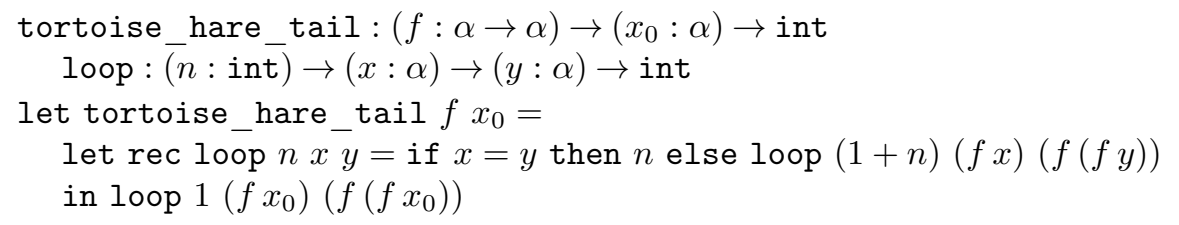

Notice that the pre-condition of cyclicity (any item of Proposition 1) is necessary otherwise the above OCaml code does not terminate and is thus incorrect. Any correctness proof must include that cyclicity pre-condition, or a stronger one.

\subsection{Goals and contributions}

The goal of this work is double:

Goal 1: functional correctness. Using purely constructive means, build fully specified Coq terms that compute a meeting point for the tortoise and the hare, with the sole pre-condition of cyclicity. Reiterate this for Floyd's and Brent's cycle finding algorithms;

Goal 2: operational correctness. Ensure that the extraction of the previous Coq terms give the corresponding standard OCaml implementations. In particular, derive the above implementations of tortoise_hare and tortoise_hare_tail by extraction.

From these two goals, trusting Coq extraction mechanism, we get the functional correctness of the standard OCaml implementations for free.

For $\mathrm{T \& H}$, solving Goal 1 can be viewed as constructing a term of type

$$
\text { th_coq }:\left(\exists \tau, 0<\tau \wedge f^{\tau} x_{0}=f^{2 \tau} x_{0}\right) \rightarrow\left\{\tau \mid 0<\tau \wedge f^{\tau} x_{0}=f^{2 \tau} x_{0}\right\}
$$

from the assumptions of a type $X$ : Type, a procedure $={ }_{X}^{?}$ for deciding equality over $X$, and a sequence given by $f: X \rightarrow X$ and $x_{0}: X$. Notice the assumption $={ }_{X}^{?}: \forall x y: X,\{x=y\}+\{x \neq y\}$ of an equality decider for $X$ that is necessary in Coq. Indeed, unlike OCaml which has a built-in polymorphic equality decider 1 Coq does (and can) not have equality deciders for every possible type.

Solving Goal 2 means that after extraction of OCaml code from th_coq, we get the same function code as tortoise_hare (resp. tortoise_hare_tail).

This paper is a companion for Coq implementations of cycle finding algorithms. The corresponding source code can be found at

$$
\text { https://github.com/DmxLarchey/The-Tortoise-and-the-Hare }
$$

The implementation involves around 3000 lines of Coq code but this does not reflect the compactness of our implementation of T\&H. Indeed, it contains Floyd's

\footnotetext{
${ }^{1}$ OCaml equality decider is partially correct, e.g. it throws exceptions on functions.
} 
and Brent's algorithms as well, and there are several accompanying files illustrating certified recursion through bar inductive predicates. To witness the conciseness of our approach, we give a standalone tail-recursive implementation of T\&H of less than 80 lines, not counting comments (see th alone.v). This project compiles under Coq 8.6 and is available under a Free Software license.

The designs of the cycle finding algorithms that we propose are all based on bar inductive predicates used as termination certificates for Coq fixpoint recursion. In Section 3 , we give a brief introduction to these predicates from a programmer's point of view and show why they are suited for solving termination problems. The corresponding Coq source code can be found in file bar.v.

In Section 4 , we present two fully specified implementations of $T \& H$, one nontail recursive and one tail-recursive. We give a detailed account of the algorithmic part of the implementation that we isolate from logical obligations. We explain how bar inductive predicates are used to separate/postpone termination proofs from algorithmic considerations. The corresponding file is tortoise hare.v.

In Section 5, we give an overview of the implementation of the full Floyd cycle finding algorithm that computes the characteristic index and period of an iterated sequence. The corresponding Coq file is floyd.v. In Section 6, we give a brief account of our implementation of Brent's period finding algorithm, in fact two implementations: one suited for binary numbers and one suited for unary numbers. The corresponding source code files are brent_bin.v and brent_una.v.

The T\&H has already been the subject of implementations in Coq [911], but under different requirements. In Section 7, we compare our development with those alternative approaches. From a constructive point of view, we analyse the pre-conditions under which correctness is established in each case.

\section{Termination using Bar Inductive Predicates}

In this section, we explain how to use bar inductive predicates [12] - a constructive and axiom-free form of bar induction ${ }^{2}$ - as termination certificates.

As explained in Section 3.2, in the context we use them (decidable terminated cases), these predicates have the same expressive power as the accessibility predicates used for well-founded recursion in Coq in the modules Wf and Wellfounded from the standard library (see also left part of Fig. 1). But we think that bar inductive predicates have several advantages over accessibility predicates:

- compared to the general accessibility predicates of [4, they do not need the simultaneous induction/recursion schemes of Dybjer [10] (not integrated in Coq so far) in case of nested/mutual recursion [17];

- unlike standard accessibility predicates (module Wf) which involve thinking about termination before implementing the algorithm, or inductively defined domain predicates (see [3] pp 427-432) which involve thinking about termination together with the algorithm, bar inductive predicates focuses on

\footnotetext{
${ }^{2}$ Conventional bar induction often requires Brouwer's thesis which precisely postulates that bar predicates are inductive.
} 


$$
\begin{array}{l|l}
\forall y, R y x \rightarrow \operatorname{Acc} y \\
\operatorname{Acc} x
\end{array} \quad \frac{T x}{\operatorname{bar} x} \quad \frac{\forall y, R x y \rightarrow \operatorname{bar} y}{\operatorname{bar} x}
$$

Fig. 1. Inductive rules for Acc and bar termination certificates.

terminated cases and recursive sub-calls so termination proofs can be separated from the algorithm.

We argue that separating/postponing the proof of termination makes the use of bar inductive predicates more versatile. At least, we hope that we illustrate our case here. Of course, a comprehensive comparison with [5] would be necessary to complete our case. The reader could be interested in recent developments that show that the method of bar inductive predicates scales well to more complicated nested/mutual recursive schemes [17].

We do not really introduce new concepts in the section. But we want to stress the links between the notion of cover-induction [8] and the notion of bar inductive predicate (e.g. inductive bars [12]). We insist on these notions because we will specialize the following generic implementation to get an "extraction friendly" Coq definition of cycle finding algorithms.

\subsection{Dependently typed recursion for bar inductive predicates}

Let us consider a type $X$, a unary relation $T: X \rightarrow$ Prop and a binary relation $R: X \rightarrow X \rightarrow$ Prop. Here are some possible intuitive interpretations of $T$ and $R$ :

$\boldsymbol{T} \boldsymbol{x}$ : the computation at point $x$ is terminated (no recursive sub-call); $\boldsymbol{R} \boldsymbol{x} \boldsymbol{y}:$ a call at point $x$ may trigger a recursive sub-call at point $y$.

We define the inductive predicate bar : $X \rightarrow$ Prop which covers points where computation is warrantied to terminate, by the two rules on the right of Fig. 1.

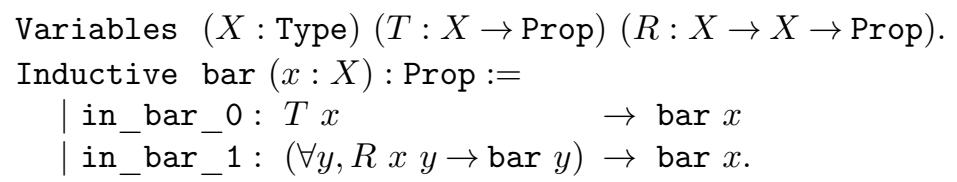

The first rule in_bar_o states that a terminated computation terminates. The second rule in_bar_ 1 states that if every recursive sub-call $y$ of $x$ terminates then so is the call at $x$. Notice that the predicate bar $x$ : Prop carries no computational content and thus cannot be used to perform computational choices. Termination is only warrantied by the bar $x$ predicate, it is not performed by it.

Hence we assume a decider term $T_{\mathrm{dec}}: \forall x,\{T x\}+\{\neg T x\}$ for terminated points. We then define bar rect, a dependently typed recursion principle for bar $x$. For this, we need the following inductions hypotheses:

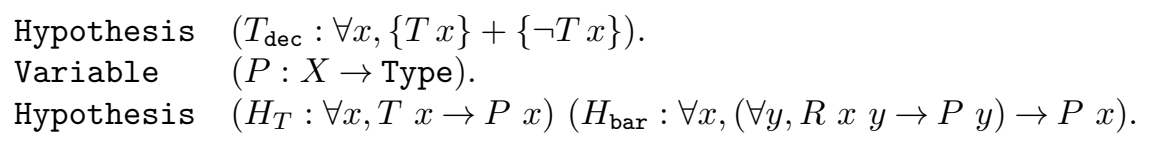


where $H_{T}$ gives the value for terminated points and $H_{\mathrm{bar}}$ combines the values of the recursive sub-calls into a value for the call itself. With these assumptions, we get the following dependently typed induction principle:

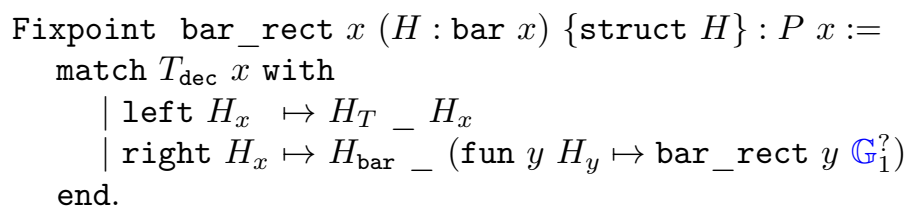

where $\mathbb{G}_{1}^{?}$ is a proof term for a logical obligation:

$$
\mathbb{G}_{1}^{?} / / \ldots, x: X, H: \operatorname{bar} x, H_{x}: \neg T x, y: X, H_{y}: R x y \vdash \operatorname{bar} y
$$

Notice that for Coq to accept such a Fixpoint definition as well-typed, one must ensure that the given proof of goal $\mathbb{G}_{1}^{?}$ is a sub-term of the term $H$ : bar $x$ because $H$ is declared as the structurally decreasing argument of this fixpoint. Hence, the first step in the proof of $\mathbb{G}_{1}^{?}$ is destruct $H$.

The above implementation of bar_rect expects the proof term of $\mathbb{G}_{1}^{?}$ to be given before the actual Fixpoint definition of bar rect. This can be mitigated with the use of the very handy refine tactic that can delay the proof obligations after the incomplete proof term is given (see bar.v for details). As a final remark concerning the term bar_rect, there are two ways of stopping a chain of recursive sub-calls: the first is obviously to reach a terminated point (i.e. $T x$ ) but the chain can also stop when there is zero recursive sub-calls (i.e. $R x y$ holds for no $y$ ). While the first condition of terminated points is decidable, the second condition of the nonexistence of recursive sub-calls is usually not decidable. Hence when using the accessibility predicate Acc (fun $u v \mapsto R v u \wedge \neg T v$ ) $x$ which mixes both $T$ and $R$ (see Theorem bar_Acc_eq_dec below), detecting the first termination condition is less natural.

\subsection{Accessibility vs. bar inductive predicates}

We show that bar inductive predicates generalize accessibility predicates defined in the Coq standard library module Wf,

$$
\begin{gathered}
\text { Theorem bar_empty_Acc_eq }(X: \text { Type })(R: X \rightarrow X \rightarrow \text { Prop) }(x: X): \\
\text { bar (fun_ } \mapsto \text { False) } R x \Longleftrightarrow \operatorname{Acc} R^{-1} x
\end{gathered}
$$

which is obvious from the rules of Fig. 1 because when $T=$ fun $\_\mapsto$ False is empty, one cannot use rule in_bar_0. Then, we show that when $T: X \rightarrow \operatorname{Prop}$ is (logically) decidable, then bar $T R$ can be encoded as an accessibility predicate:

$$
\begin{aligned}
& \text { Theorem bar_Acc_eq_dec } X(T: X \rightarrow \text { Prop) }(R: X \rightarrow X \rightarrow \text { Prop) }: \\
& \quad(\forall x, T x \vee \neg T x) \rightarrow \forall x \text {, bar } T R x \Longleftrightarrow \text { Acc (fun } u v \mapsto R v u \wedge \neg T v) x
\end{aligned}
$$

From our point of view, the advantage of bar over Acc is that they keep the two forms of termination separate ( $x$ is terminated by $T$ vs. $x$ generates no recursive sub-call), making them easier to reason or compute with. Moreover, using 
Acc incites at using only well-founded relations (or even decreasing measures) whereas bar focuses on terminated points/recursive calls and thus can be used more freely as exemplified in Sections 4 and 5 .

\subsection{Constructive epsilon via bar inductive predicates}

As a first illustration of using bar inductive predicates, we show how to implement Constructive Indefinite Ground Description defined in the standard library module ConstructiveEpsilon.

$$
\begin{aligned}
& \text { Theorem Constructive_Epsilon }(Q: \text { nat } \rightarrow \text { Prop }): \\
& \qquad(\forall n,\{Q n\}+\{\neg Q n\}) \rightarrow(\exists n, Q n) \rightarrow\{n: \text { nat } \mid Q n\} .
\end{aligned}
$$

We instantiate bar_rect with $(T:=Q),(R x y:=\mathrm{S} x=y)$ and $\left(P \_:=\right.$ $\{x \mid Q x\})$. We only have to transform the termination certificate $\exists n, Q n$ into a bar inductive predicate at the purely logical/Prop level. For this, we show $(\exists n, Q n) \rightarrow$ bar $Q R$ 0: from $Q n$ deduce bar $Q R n$ using in bar_0 and then bar $Q R(n-1), \ldots$ down to bar $Q R 0$ by descending induction ${ }^{3}$ using in_bar_1.

Notice that using the previous development, we can already implement the functional specification of the T\&H algorithm:

$$
\text { th_min }:\left(\exists \tau, 0<\tau \wedge f^{\tau} x_{0}=f^{2 \tau} x_{0}\right) \rightarrow\left\{\tau \mid 0<\tau \wedge f^{\tau} x_{0}=f^{2 \tau} x_{0}\right\}
$$

by application of Constructive_Epsilon with $\left(Q n:=0<n \wedge f^{n} x_{0}=f^{2 n} x_{0}\right)$. Indeed, such $Q:$ nat $\rightarrow$ Prop is computationally decidable as both $<:$ nat $\rightarrow$ nat $\rightarrow$ Prop and $=_{X}: X \rightarrow X \rightarrow$ Prop are computationally decidable $4^{4}$

However, this approach will not give us the operational specification of $\mathrm{T \& H}$ because this implementation of th_min using Constructive_Epsilon extracts into the inefficient unbounded minimization algorithm on the right-hand side (see also th_min.v). There, $\mu$ min corre-

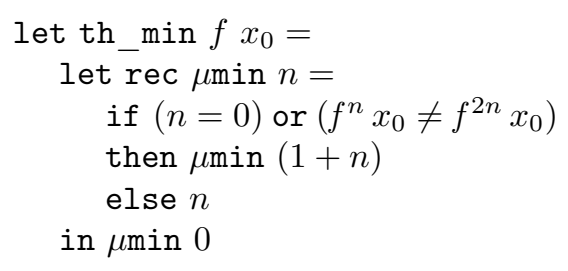
sponds to unbounded minimization. This th_min program recomputes $f^{n} x_{0}$ and $f^{2 n} x_{0}$ for each value of $n$ before a cycle is detected, making it really inefficient.

\section{The Tortoise and the Hare via Bar Inductive Predicates}

In this section, we use the methodology of Section 3 (i.e. termination via bar inductive predicates) to design a fully specified implementation of the $\mathrm{T} \& \mathrm{H}$ algorithm that satisfies Goals 1 and 2 of Section 2.2. We could use bar_rect to implement this algorithm but we do not use it directly. Indeed, we want to finely control the computational content of our terms so that we can extract the

\footnotetext{
${ }^{3}$ descending induction is implemented by nat_rev_ind in file utils.v
}

${ }^{4}$ for $=_{X}$, this is precisely the assumption of the $={ }_{X}^{?}$ equality decider. 


$$
\frac{x=y}{\operatorname{bar}_{\mathrm{th}} x y} \quad \frac{\operatorname{bar}_{\mathrm{th}}(f x)(f(f y))}{\operatorname{bar}_{\mathrm{th}} x y} \quad \mid \frac{x=y}{\operatorname{bar}_{\mathrm{t} 1} i x y} \quad \frac{\operatorname{bar}_{\mathrm{t} 1}(\mathrm{~S} i)(f x)(f(f y))}{\operatorname{bar}_{\mathrm{t} 1} i x y}
$$

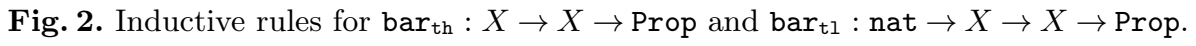

expected OCaml code accurately. However, we will mimic the implementation of bar_rect several times. The corresponding file for this section is tortoise_hare.v.

The T\&H detects potential cycles in the iterated values of an endo-function $f: X \rightarrow X$. As explained in Section 2.2, for the remaining of this section, we assume the following pre-conditions for the hare to recapture the tortoise:

$$
\begin{array}{ll}
\text { Variables } & (X: \text { Type })\left(={ }_{X}^{?}: \forall x y: X,\{x=y\}+\{x \neq y\}\right) \\
& (f: X \rightarrow X)\left(x_{0}: X\right)\left(H_{0}: \exists \tau, 0<\tau \wedge f^{\tau} x_{0}=f^{2 \tau} x_{0}\right) .
\end{array}
$$

that is a type $X$ with an equality decider $={ }_{X}^{?}$, a sequence $f$ starting at point $x_{0}$ satisfying a cyclicity assumption $H_{0}$ (see Proposition 1). These pre-conditions are not minimal for establishing the correctness of $T \& \mathrm{H}^{5}$ but we do think they are general enough to accommodate most use cases of $\mathrm{T} \& \mathrm{H}$.

\subsection{A non-tail recursive implementation}

Let us start with the non-tail recursive implementation of $\mathrm{T} \& \mathrm{H}$, as is done in the OCaml code of tortoise hare (see Section 2.1). We define a bar inductive predicate which will be used as termination certificate for the main loop tort_hare_rec. Compared to the generic inductive definition of bar of Section 3, bar $_{\text {th }}$ is a binary (instead of unary) bar predicate specialized with $(T x y:=x=y)$ and $(R x y u v:=u=f x \wedge v=f(f y))$ :

$$
\begin{aligned}
& \text { Inductive } \operatorname{bar}_{\text {th }}(x y: X) \text { : Prop := } \\
& \text { in_bar_th_0: } x=y \quad \rightarrow \operatorname{bar}_{\text {th }} x y \\
& \text { in_bar_th_1: } \operatorname{bar}_{\text {th }}(f x)(f(f y)) \rightarrow \text { bar }_{\text {th }} x y
\end{aligned}
$$

This definition matches the inductive rules of Fig. 2 (left part). We define a fully specified Coq term tort_hare_rec mimicking both the OCaml code of Section 2.1 (with the addition of a termination certificate of type $\operatorname{bar}_{\operatorname{th}} x y$ ) and the code of bar_rect of Section 3 .

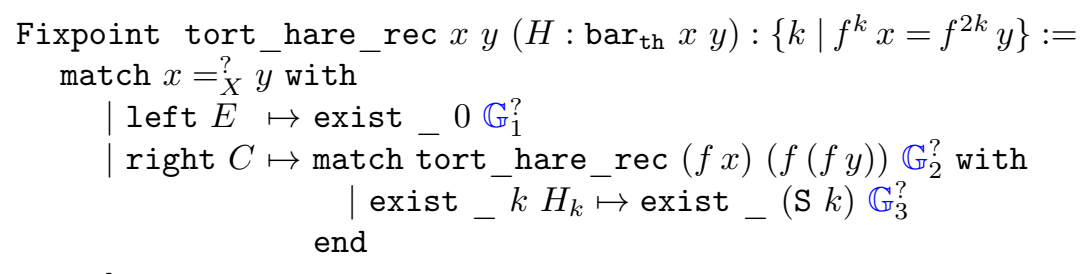

end.

\footnotetext{
$\overline{{ }^{5} \text { see } \overline{\text { th_rel.v }}}$ where an arbitrary decidable relation $R: X \rightarrow X \rightarrow$ Prop replaces $={ }_{X}$.
} 
where $\mathbb{G}_{1}^{?}, \mathbb{G}_{2}^{?}$ and $\mathbb{G}_{3}^{?}$ are three proof terms of the following types:

$$
\begin{array}{lll}
\mathbb{G}_{1}^{?} & / / & \ldots, E: x=y \vdash f^{0} x=f^{2.0} y \\
\mathbb{G}_{2}^{?} & / / & \ldots, C: x \neq y, H: \operatorname{bar}_{\mathrm{th}} x y \vdash \operatorname{bar}_{\mathrm{th}}(f x)(f(f y)) \\
\mathbb{G}_{3}^{?} & / / & \ldots, H_{k}: f^{k}(f x)=f^{2 k}(f(f y)) \vdash f^{\mathrm{s} k} x=f^{2(\mathrm{~s} k)} y
\end{array}
$$

These can be established before the Fixpoint definition of tort_hare_rec or else (preferably), using the Coq refine tactic, after the statement of the computational part of tort_hare_rec, as remaining logical obligations (see tortoise_hare.v for exact Coq code). Recall that the termination certificate $H$ must structurally decrease, i.e. the proof term for $\mathbb{G}_{2}^{?}$ must be a sub-term of $H$.

We can now define tortoise_hare by calling tort_hare_rec but we need to provide a termination certificate:

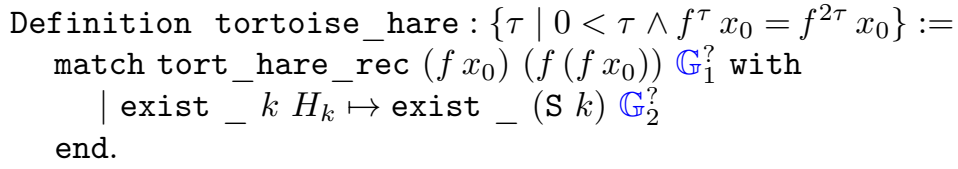

There are two remaining logical obligations, $\mathbb{G}_{1}^{?}$ being the termination certificate:

$$
\begin{aligned}
& \mathbb{G}_{1}^{?} \quad / / \ldots, H_{0}: \exists \tau, 0<\tau \wedge f^{\tau} x_{0}=f^{2 \tau} x_{0} \vdash \operatorname{bar} \operatorname{th}\left(f x_{0}\right)\left(f\left(f x_{0}\right)\right) \\
& \mathbb{G}_{2}^{?} / / \ldots, H_{k}: f^{k}\left(f x_{0}\right)=f^{2 k}\left(f\left(f x_{0}\right)\right) \vdash 0<\mathrm{S} k \wedge f^{\mathrm{S} k} x_{0}=f^{2(\mathrm{~S} k)} x_{0}
\end{aligned}
$$

We prove $\mathbb{G}_{1}^{?}$ as follows: from $H_{0}$, we (non-computationally) deduce $m$ such that $0<m$ and $f^{m} x_{0}=f^{2 m} x_{0}$. Using in_bar_th_0 we immediately get $\operatorname{bar}_{\text {th }}\left(f^{m} x_{0}\right)\left(f^{2 m} x_{0}\right)$. Then using in_bar_th_1 repeatedly from $m, m-1, \ldots$ down to 1 we get $\operatorname{bar}_{\text {th }}\left(f^{1} x_{0}\right)\left(f^{2} x_{0}\right)$. $\mathbb{G}_{2}^{?}$ is obtained by trivial computations over nat using the $f_{-}$equal/omega tactics.

The Coq command Recursive Extraction tortoise_hare produces the corresponding OCaml code of Section 2.1 except that the OCaml type int is replaced with nat and the OCaml built-in equality decider is replaced with (a to be provided implementation of) $=?$ ?

\subsection{A tail-recursive implementation}

Now we proceed with the tail-recursive implementation of T\&H. We define a ternary bar inductive predicate corresponding to the recursive call of the loop in the OCaml code of tort_hare_tail in Section 2.1.

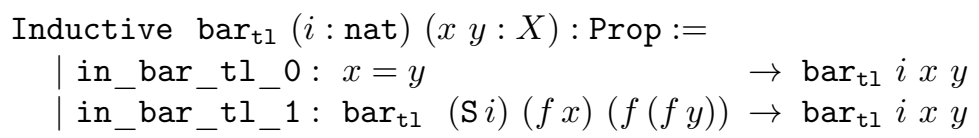

the corresponding inductive rules being described in Fig. 2 (right part). Then we can define the internal loop of tort_hare_tail by a (local) fixpoint over 
the fourth argument of type $\operatorname{bar}_{\mathrm{tl}} i x y$ :

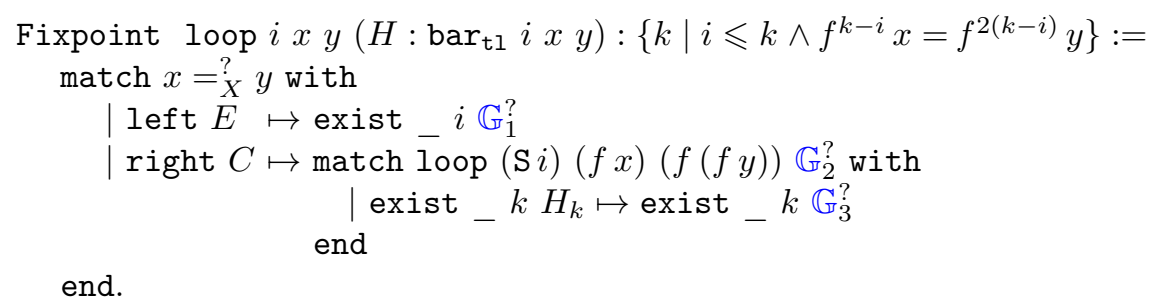

where $\mathbb{G}_{1}, \mathbb{G}_{2}^{?}$ and $\mathbb{G}_{3}^{?}$ are three proof terms of the following types:

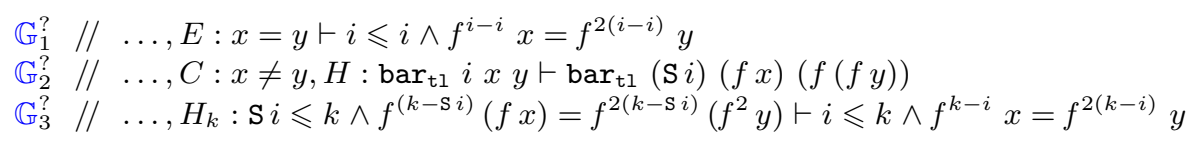

and $\mathbb{G}_{2}^{?}$ is the termination certificate and must be a sub-term of $H$. Then we proceed with the implementation of tortoise_hare_tail which calls loop:

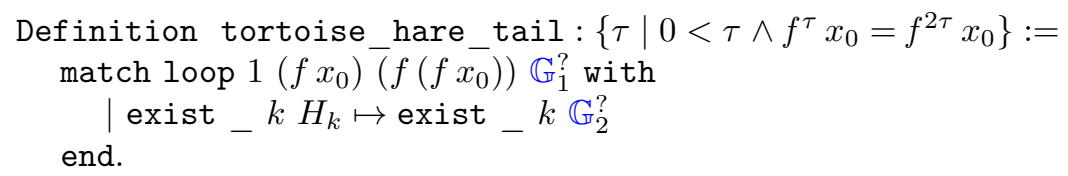

We must provide a termination certificate $\mathbb{G}_{1}^{?}$ and establish the specification $\mathbb{G}_{2}^{\text {? }}$ :

$$
\begin{aligned}
& \mathbb{G}_{1}^{?} / / \ldots, H_{0}: \exists \tau, 0<\tau \wedge f^{\tau} x_{0}=f^{2 \tau} x_{0} \vdash \operatorname{bar}_{\mathrm{t} 1} 1\left(f x_{0}\right)\left(f\left(f x_{0}\right)\right) \\
& \mathbb{G}_{2}^{?} / / \quad \ldots, H_{k}: 1 \leqslant k \wedge f^{k-1}\left(f x_{0}\right)=f^{2(k-1)}\left(f\left(f x_{0}\right)\right) \vdash 0<k \wedge f^{k} x_{0}=f^{2 k} x_{0}
\end{aligned}
$$

$\mathbb{G}_{1}^{?}$ is proved by descending induction much like what is done in the non-tail recursive case and $\mathbb{G}_{2}^{?}$ is quite trivial to obtain using the $f$ _equal/omega tactics.

The extracted OCaml code corresponds to the tortoise_hare_tail implementation of Section 2.1. The file th_alone.v contains a standalone implementation of tortoise_hare_tail in less than 80 lines, not counting comments.

\section{$5 \quad$ Floyd's Cycle Finding Algorithm in Coq}

In this section, we give an overview of Floyd's index and period finding algorithm as implemented in the file floyd.v. It has the same pre-conditions as the T\&H algorithms of Section 4

$$
\begin{array}{ll}
\text { Variables } & (X: \text { Type })\left(={ }_{X}^{?}: \forall x y: X,\{x=y\}+\{x \neq y\}\right) \\
& (f: X \rightarrow X)\left(x_{0}: X\right)\left(H_{0}: \exists \tau, 0<\tau \wedge f^{\tau} x_{0}=f^{2 \tau} x_{0}\right) .
\end{array}
$$

It does not only finds a meeting point for the tortoise and the hare but computes the characteristic pair of values $(\lambda, \mu)$ of the cycle which satisfy the predicate cycle_spec with the following body:

$$
\begin{aligned}
& \text { Definition cycle_spec }(\lambda \mu \text { : nat) : Prop }:= \\
& 0<\mu \wedge f^{\lambda} x_{0}=f^{\lambda+\mu} x_{0} \wedge \forall i j, i<j \rightarrow f^{i} x_{0}=f^{j} x_{0} \rightarrow \lambda \leqslant i \wedge \mu \operatorname{div}(j-i) .
\end{aligned}
$$


where div represents the divisibility order over nat (i.e. $d$ div $n$ means $\exists q, n=$ $q d)$. The index $\lambda$ is such that $f^{\lambda}\left(x_{0}\right)$ is the entry point of the cycle and $\mu>0$ is the period (or length) of the cycle. The third conjunct $(\forall i j, i<j \rightarrow \ldots)$ states that any (non-empty) cycle $i \rightsquigarrow j$ occurs after $\lambda$ and has a length divisible by $\mu$.

Hence under the above pre-conditions, Floyd's algorithm has the functional specification floyd_find_cycle $:\{\lambda:$ nat $\&\{\mu$ : nat $\mid$ cycle_spec $\lambda \mu\}\}$. The operational specification is simply that the Coq term extracts to a standard OCaml implementation derived from [1].floyd_find_cycle is implemented as the combination of three sub-terms, floyd_meeting_pt which first computes a meeting point for the tortoise and the hare, then floyd_index that computes the index and finally floyd period that computes the period. We describe these three sub-terms in specific sub-sections, each sub-section potentially having its own set of additional pre-conditions, mimicking Coq sectioning mechanism. For each of these terms, we use a tailored bar inductive predicate to ensure termination under the corresponding pre-conditions.

\subsection{Computing a meeting point}

The term floyd meeting pt needs no further pre-conditions. We use the inductive $\operatorname{bar}_{\mathrm{th}}: \bar{X} \rightarrow X \rightarrow$ Prop of Fig. 2 as termination certificate, the same that we used for the non-tail recursive tortoise_hare implementation. However, we do get a tail-recursive term here because we only compute a meeting point, not its index in the sequence as in the bar $_{\mathrm{t} 1} /$ tortoise_hare_tail case.

$$
\begin{aligned}
& \text { Let bar_th_meet: } \forall x y, \operatorname{bar}_{\text {th }} x y \rightarrow\left\{c: X \mid \exists k, c=f^{k} x \wedge c=f^{2 k} y\right\} . \\
& \text { Definition floyd_meeting_pt : }\left\{c \mid \exists \tau, 0<\tau \wedge c=f^{\tau} x_{0} \wedge c=f^{2 \tau} x_{0}\right\} .
\end{aligned}
$$

We define bar_th_meet as a local fixpoint using the same technique as in the bar $_{\text {th }} /$ tortoise_hare case. Then, we show $H_{0}^{\prime}: \operatorname{bar}_{\text {th }}\left(f x_{0}\right)\left(f\left(f x_{0}\right)\right)$ as a consequence of $\bar{H}_{0}$ and we derive floyd_meeting_pt from the following instance bar_th_meet $\left(f x_{0}\right)\left(f\left(f x_{0}\right)\right) H_{0}^{\prime}$.

\subsection{Computing the index}

The term floyd_index uses the post-condition of floyd_meeting_pt as a further pre-condition, i.e. a meeting point $c$ for the tortoise and the hare. We use the predicate bar $_{\text {in }}$ : nat $\rightarrow X \rightarrow X \rightarrow$ Prop of Fig. 3 as termination certificate.

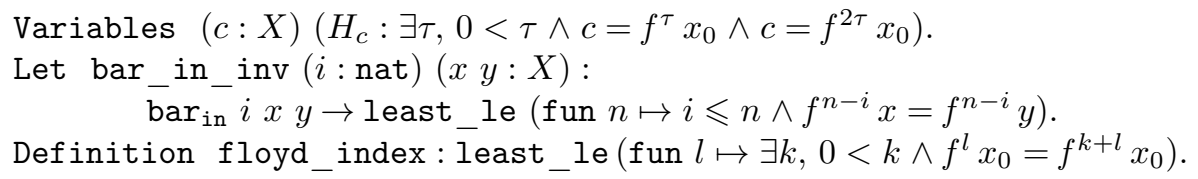

We define bar_in_inv as a local fixpoint where least_le $P$ is the least ${ }^{6}$ $n$ : nat which satisfies $P n$. We show $H_{c}^{\prime}$ : $\operatorname{bar}_{\text {in }} 0 x_{0} c$ as a consequence of $H_{c}$ and we derive floyd_index from the following instance bar_in_inv $0 x_{0} c H_{c}^{\prime}$.

\footnotetext{
${ }^{6}$ least for the natural order $\leqslant$ over nat.
} 


$$
\frac{x=y}{\operatorname{bar}_{\mathrm{in}} i x y} \quad \frac{\operatorname{bar}_{\mathrm{in}}(\mathrm{S} i)(f x)(f y)}{\operatorname{bar}_{\mathrm{in}} i x y} \quad \mid \frac{c=y}{\operatorname{bar}_{\mathrm{pe}} i y} \quad \frac{\operatorname{bar}_{\mathrm{pe}}(\mathrm{S} i)(f y)}{\operatorname{bar}_{\mathrm{pe}} i y}
$$

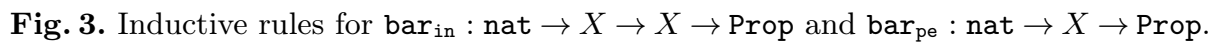

\subsection{Computing the period}

The further pre-condition of floyd_period is a point $c$ which belongs to a (nonempty) cycle, a direct consequence of the post-condition of floyd_meeting_pt. Termination is certified by the predicate bar $_{\mathrm{pe}}:$ nat $\rightarrow X \rightarrow$ Prop of Fig. 3 .

$$
\begin{aligned}
& \text { Variables }(c: X)\left(H_{c}: \exists k, 0<k \wedge c=f^{k} c\right) \text {. } \\
& \text { Let bar_pe_inv } i x: \operatorname{bar}_{\text {pe }} i x \rightarrow \text { least_le }\left(\text { fun } n \mapsto i \leqslant n \wedge x=f^{n-i} y\right) . \\
& \text { Definition floyd_period : least_div (fun } \left.n \mapsto 0<n \wedge c=f^{n} c\right) .
\end{aligned}
$$

We define bar_pe_inv as a local fixpoint. We prove $H_{c}^{\prime}: \operatorname{bar}_{\mathrm{pe}} 1(f c)$ using $H_{c}$ and we get floyd_period from the following instance bar_pe_inv 1 ( f c $) H_{c}^{\prime}$. Here, least_div $P$ is the least $n$ s.t. $P n$ for the divisibility order div.

\subsection{Gluing all together}

We finish with the term floyd_find_cycle:

$$
\text { Definition floyd_find_cycle: }\{\lambda \text { : nat } \&\{\mu \text { : nat } \mid \text { cycle_spec } \lambda \mu\}\} \text {. }
$$

It needs no further pre-conditions than those given at the beginning Section 5 . It is composed of the successive applications of floyd_meeting_pt, floyd_index and floyd_period where the post-condition of floyd_meeting_pt serves as input for the extra pre-conditions of floyd_index and floyd_period. We conclude with a short proof that the computed index and period satisfy cycle_spec. The extracted OCaml program corresponds to the following code with the same remarks regarding $={ }_{X}^{?}$ and nat/int as with tortoise_hare from Section 4.1 .

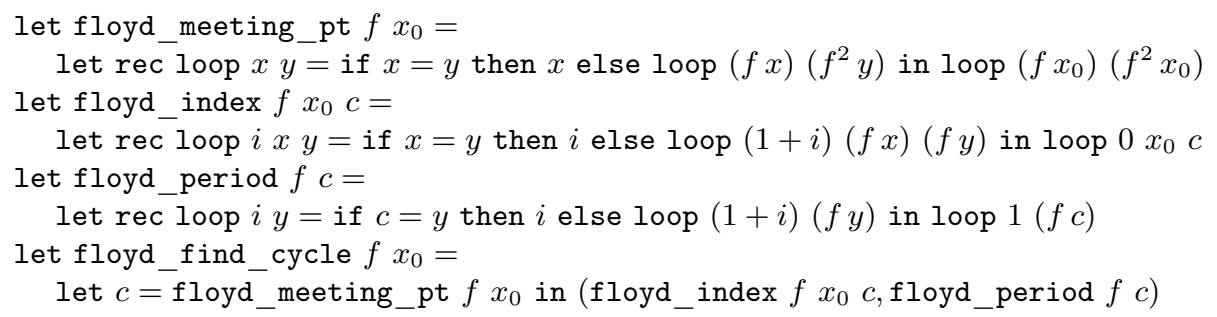

\section{Brent's Period Finding Algorithm}

In the file brent_bin.v, we propose a correctness proof of Brent's algorithm in the same spirit as what was done for Floyd's cycle finding algorithm of Section 5 . 
Brent's algorithm [6] only computes the period $\mu$ of the cycle. The index $\lambda$ can be computed afterwards by using two tortoises separated by $\mu$ steps. Brent's algorithm is more efficient than T\&H: it can be proved that a run of Brent's algorithm on $\left(f, x_{0}\right)$ always generates less calls to $f$ than a run of the T\&H (or Floyd's cycle finding) algorithm on the same input (see [14, Sect. 7.1.2).

In this section, we just describe the functional specification and the operational specification (i.e. the extracted OCaml code) of Brent's algorithm of which we propose two implementations. The first one in file brent_bin.v is suited for a binary representation of natural numbers, but it is not efficient with unary natural numbers. The other implementation in file brent_una.v is also efficient on unary natural numbers such as those of type nat.

Given a type $X$ : Type, an equality decider $={ }_{X}^{?}: \forall x y: X,\{x=y\}+$ $\{x \neq y\}$, input values $f: X \rightarrow X$ and $x_{0}: X$, and a cycle existence certificate (see Proposition 1), Brent's algorithm computes a $\mu$ satisfying the specification period_spec : $\forall \mu$ : nat, Prop with the following body

$$
0<\mu \wedge\left(\exists \lambda, f^{\lambda} x_{0}=f^{\lambda+\mu} x_{0}\right) \wedge \forall i j, i<j \rightarrow f^{i} x_{0}=f^{j} x_{0} \rightarrow \mu \operatorname{div}(j-i)
$$

that is, it computes the period of the cycle. The term brent_bin extracts to something close to the following OCaml code:

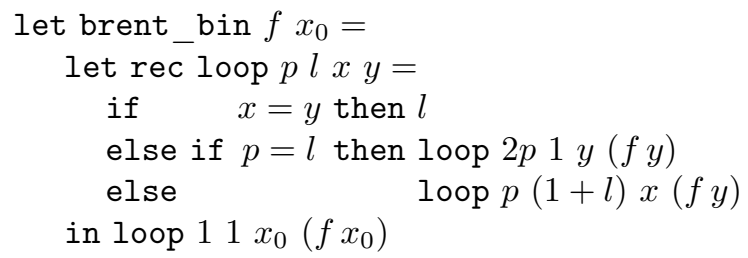

where int is replaced with nat, $(x=y)$ with $\left(x=?_{X}^{?} \quad y\right)$ and $(p=l)$ with (eq_nat_dec $x y$ ). However this code is not optimal with a unary representation of numbers such as nat: in particular $2 p$ and $p=l$ are slow (linear) to compute.

To get a more efficient implementation, one should either use a binary representation of numbers or switch to brent_una which has the same specification as the binary version but extracts to the following OCaml code:

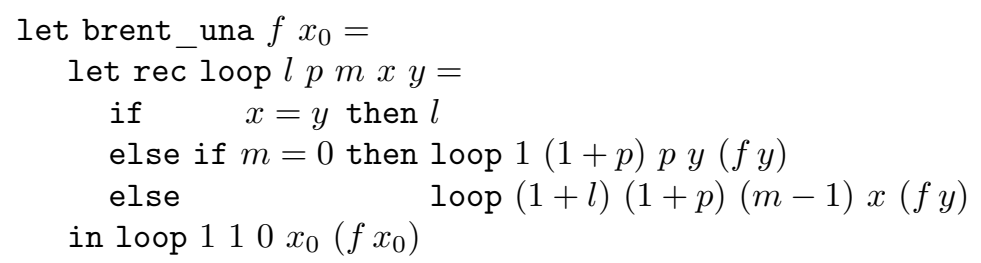

This code is much better suited for unary numbers. In particular, $m=0$ and $m-1$ are computed via pattern-matching on $m$ in constant time.

\section{Correctness by Extraction and Related Works}

Correctness is a property of programs with respect to a given specification. As trivial as this remark may seem, it is important to keep it in mind because the 
purpose of extraction is to erase the logical content of Coq programs and to keep only their computational content: specifications are erased by extraction. One cannot claim that a program extract $(t)$ is correct just because it has been extracted from a Coq term $t: T$. The correctness property is only ensured with respect to the particular specification $T$ that (by the way) had just been erased.

\subsection{Correctness of the Tortoise and the Hare}

We illustrate this critical aspect of extraction on the non-tail recursive OCaml implementation of $\mathrm{T \& H}$. The type of tortoise_hare is

$$
\begin{gathered}
\text { Definition tortoise_hare }\{X\}(=? \stackrel{?}{X}: \forall x y: X,\{x=y\}+\{x \neq y\}) f x_{0}: \\
\left(H_{0}: \exists \tau, 0<\tau \wedge f^{\tau} x_{0}=f^{2 \tau} x_{0}\right) \rightarrow\left\{\tau: \text { nat } \mid 0<\tau \wedge f^{\tau} x_{0}=f^{2 \tau} x_{0}\right\} .
\end{gathered}
$$

as reported from Section 4 . The pre-conditions of this specification are all the logical properties of the input parameters, i.e. the fact that $={ }_{X}^{?}$ is an equality decider for $X$ and the cyclicity property $H_{0}$. The post-condition is the fact that $\tau$ is a meeting index for the fabulous animals. By extraction, the OCaml code tortoise_hare of Section 2.1 is correct w.r.t. to this specification. In the extracted program however, the pre-conditions on $={ }_{X}^{?}$ and of cyclicity, and the post-conditions $0<\tau$ and $f^{\tau} x_{0}=f^{2 \tau} x_{0}$ have disappeared.

Now consider this "alternative" (and cheating) implementation of T\&H:

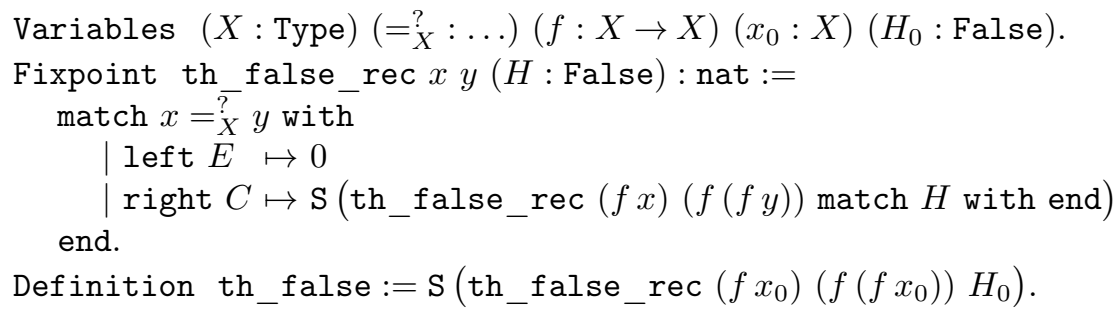

The pre-conditions for th_false are the same as those of tortoise_hare except that cyclicity has been replaced with absurdity $\left(H_{0}:\right.$ False). The postcondition has been erased. Yet, up to renaming, extraction of OCaml code from th_false and from tortoise_hare yields the very same program. So the OCaml program tortoise hare/th false is correct w.r.t. two very different specifications: one is useful (cyclicity) and one is useless (absurdity).

The file infinite_loop.ml contains th_false and gives other illustrations of abuses of extraction in absurd contexts. As a conclusion, before using or running an extracted algorithm, one should first check for assumptions in the specification using the Coq command Print Assumptions which lists all the potentially hidden pre-conditions such as axioms or parameters which are usually not displayed in the types of terms in Coq to avoid bloating them.

\subsection{Comparison with Related Works}

Given the stunning simplicity of $T \& H$, our implementation is hardly the first attempt at certifying this algorithm. As for Coq, may be there are others, but 
we are aware of two previous developments. One (unpublished) proof by J.C. Filliâtre [11] and another, more recent, by J.F. Dufourd [9].

Illustrating how Hilbert's $\epsilon$-operator could be used to manage partial functions within Coq was the main goal of his implementation [11] (private communication with J.C. Filliâtre); and the correctness of T\&H was not really a goal of that project. The use of epsilon/epsilon_spec to axiomatize Hilbert's $\epsilon$-operator is, from a constructive point of view, our main criticism against that implementation. Hence, while it is true that the term find_cycle of fillia_orig.v extracts to some OCaml code very similar to tortoise_hare_tail of Section 2.1, the corresponding specification has stronger pre-conditions than our own. Following the observations on correctness of Section 7.1. even if erased by extraction, Hilbert's $\epsilon$-operator is still a pre-condition and a particularly strong form of the axiom of choice. Anything that exists can be reified with this operator. While not necessarily contradictory in itself, that kind of axiom is incompatible with several extensions of Coq [7]. We do not think it can be accepted from a constructive point of view because admitting the $\epsilon$-operator allows to write non-recursive functions in Coq. We suggest the interested reader to consult the file collatz.v to see how the $\epsilon$-operator "solves" the Halting problem or the Collatz problem [16]. It is our claim that although find_cycle implements some correctness property of $\mathrm{T \& H}$, the pre-conditions under which this correctness is achieved cannot be constructively or computationally satisfied. In the file fillia_modif.v, we propose a modified version of [11] where the $\epsilon$-operator is replaced with Constructive_Epsilon from Section 3.3. This requires in-depth changes, in particular, on the induction principle used to ensure termination. We additionally mention a more recent Isabelle/HOL proof of P. Gammie [13] which seems to reuse the technique of J.C. Filliâtre.

The work of J.F. Dufourd [9] is based on different assumptions and the correctness proof of $\mathrm{T \& H}$ that he obtains derives from a quite large library on functional orbits over finite domains of around 20000 lines of code. The preconditions do not assume cyclicity. Instead there is a stronger assumption of finiteness of the domain, from which cyclicity can be derived using the pigeon hole principle (PHP). Admittedly, this finiteness assumption is not unreasonable, even constructively: most use cases of cycle finding algorithms occur over finite domains. However, it is our understanding that Pollard's rho algorithm [18] is run on a finite domain of unkown (i.e. non-informative) cardinality. As far as we can understand J.F. Dufourd's code, his inductive proofs are cardinality based. Hence an informative bound on the cardinal of the domain is likely a precondition for correctness. Thus, we think that his correctness proof might not be applicable to Pollard's rho algorithm (non-informative finiteness, see below).

On the other hand, modifying our specification of tortoise_hare_tail to replace cyclicity by finiteness involves the PHP (see php.v). In that case, finiteness could be expressed as the predicate $(\exists l:$ list $X, \forall x: X$, In $x l)$ which postulates the non-informative existence of a list covering all the type $X$. Hence we would obtain a specification compatible with the context of Pollard's rho algorithm. This short development can be found in th_finite.v 


\section{References}

1. Cycle detection - Wikipedia, The Free Encyclopedia

2. Aumasson, J.P., Meier, W., Phan, R., Henzen, L.: The Hash Function BLAKE. Springer Publishing Company, Incorporated (2014)

3. Bertot, Y., Castéran, P.: Interactive Theorem Proving and Program Development Coq'Art: The Calculus of Inductive Constructions. Texts in Theoretical Computer Science. An EATCS Series, Springer (2004)

4. Bove, A., Capretta, V.: Modelling general recursion in type theory. Math. Structures Comput. Sci. 15(4), 671-708 (2005)

5. Bove, A., Krauss, A., Sozeau, M.: Partiality and Recursion in Interactive Theorem Provers - An Overview Math. Structures Comput. Sci. 26(1), 38-88 (2016)

6. Brent, R.P.: An improved Monte Carlo factorization algorithm BIT Numerical Mathematics 20(2), 176-184 (Jun 1980)

7. Castéran, P.: Utilisation en Coq de l'opérateur de description (2007), http:// jfla.inria.fr/2007/actes/PDF/03_casteran.pdf

8. Coen, C.S., Valentini, S.: General Recursion and Formal Topology In: PAR-10, Partiality and Recursion in Interactive Theorem Provers. EPiC Series in Computing, vol. 5, pp. 72-83. EasyChair (2012)

9. Dufourd, J.F.: Formal study of functional orbits in finite domains Theoret. Comput. Sci. 564, 63-88 (2015)

10. Dybjer, P.: A general formulation of simultaneous inductive-recursive definitions in type theory. J. Symb. Log. 65(2), 525-549 (2000)

11. Filliâtre, J.C.: Tortoise and the hare algorithm (2007), https://github.com/ coq-contribs/tortoise-hare-algorithm

12. Fridlender, D.: An Interpretation of the Fan Theorem in Type Theory. In: TYPES'98 Selected Papers. Lecture Notes in Comput. Sci., vol. 1657, pp. 93-105. Springer (1999)

13. Gammie, P.: The Tortoise and Hare Algorithm (2015), https://www.isa-afp. org/entries/TortoiseHare.html

14. Joux, A.: Algorithmic Cryptanalysis. Cryptography and Network Security, Chapman \& Hall/CRC (2009)

15. Knuth, D.E.: The Art of Computer Programming, Volume 2: Seminumerical Algorithms. Addison-Wesley Longman Publishing Co., Inc., Boston, MA, USA (1997)

16. Lagarias, J.: The Ultimate Challenge: The $3 x+1$ Problem. American Mathematical Society (2010)

17. Larchey-Wendling, D., Monin, J.F.: Simulating Induction-Recursion for Partial Algorithms. In: TYPES 2018, Braga, Portugal (2018)

18. Pollard, J.M.: A monte carlo method for factorization. BIT Numerical Mathematics 15(3), 331-334 (Sep 1975) 